\title{
Design of QoS-Aware Energy-Efficient Fiber-Wireless Access Networks
}

\author{
G. Schütz and N. Correia
}

\begin{abstract}
Energy-efficient network design has recently become a very important topic because of the energy cost increases in service providers' infrastructures. This is of particular importance in access networks because of the growing demand for digital traffic by end users. Here we address the challenge of reducing the energy consumption of fiber-wireless (FiWi) access networks, which use both optical and radio frequency technologies to provide high bandwidth and ubiquity for end-user applications, while keeping delay under a threshold. Our goal is to find optimal sleep mode schedulings that allow energy consumption to be reduced while keeping packet delay acceptable. For this purpose a mathematical formalization and an algorithm are developed. The results show that the proposed approach is able to reduce the average packet delay, with negligible energy cost increases, in many scenarios, besides being computationally efficient and scalable. The proposed approach may, therefore, serve as a basis for planning and design of quality-of-service-aware energy-efficient FiWi access networks.
\end{abstract}

\section{INTRODUCTION}

$\mathbf{E}$ nergy-efficient communication networks have become a quite important research topic over recent years not only due to the widespread sensitivity to ecological issues but also due to the continuously increasing energy costs in service providers' infrastructures [1]. This is of particular importance in access networks since Internet demands seem not to slow down. Under such a scenario, energy efficiency becomes an important criterion when planning access networks.

A common approach to reduce energy consumption in communication networks is to allow devices to switch to sleep mode when traffic intensity is low [2,3]. By adequately putting to sleep and waking up devices, according to traffic levels, significant reductions in energy consumption can be achieved without sacrificing network utilization. However, in bursty traffic scenarios, which is the case of the Internet, the degradation of the quality of service (QoS) experienced by users may be intolerable as packets must be kept in queues or rerouted to other possibly overloaded nodes. Thus, maximization of energy savings should be constrained by predefined performance parameters such as delay.

In this paper we focus on access networks, the "last mile" of telecommunication networks connecting the central office (CO) to residential and business customers [4]. The access section comprises a large part of the Internet and contains a large number of active elements, where traffic aggregation occurs to a lesser extent compared to the core, becoming a major energy consumer [5]. Here we address the challenge of reducing the energy consumption of fiber-wireless (FiWi) access networks while keeping delay under a threshold. FiWi access networks have been proposed as flexible and cost-effective solutions because fiber is provided as far as possible, from the $\mathrm{CO}$ to the end users, and then wireless is used at the front-end where fiber can be difficult to install [6]. At the back-end, a passive optical network (PON) is usually used because of low maintenance, high reliability and low power consumption [2]. PONs consist of point-to-multipoint optical structures where an optical line terminal (OLT) at the CO serves many optical network units (ONUs) near the users. This is a time division multiplexing (TDM) shared optical medium where the downstream signal is broadcasted to all ONUs. For the upstream, the ONUs are kept synchronized in a TDM fashion so that their signals can be joined, ensuring a collision-free medium.

Within PONs, the ONUs are the devices where a reduction in energy expenditure would have most impact on network energy efficiency. These devices are not only higher in number, when compared with the OLTs for example, but also aggregate less traffic. Besides this, the downstream traffic of current PONs is broadcasted to all ONUs even though some ONUs have not requested it [2]. The ONU simply discards data that are not destined for it, meaning that significant wastage of energy exists. To avoid such wastage of energy, ONUs can be switched to sleep mode when they have no traffic to send or receive. During sleep periods, any data arriving at the OLT or ONUs will be buffered.

Energy efficiency is also of paramount importance in wireless networks, and sensor networks in particular, because of the need to save battery power [7]. In the case of FiWi access networks many routes, and many optical access points, are available from each wireless router up to the optical link. The routes to be adopted should be the ones that allow devices (wireless or optical) to switch to standby/sleep mode, improving the overall network energy efficiency. Because of such flexibility, FiWi access architectures not only become cost-effective solutions but also bring great opportunities for energy savings. 


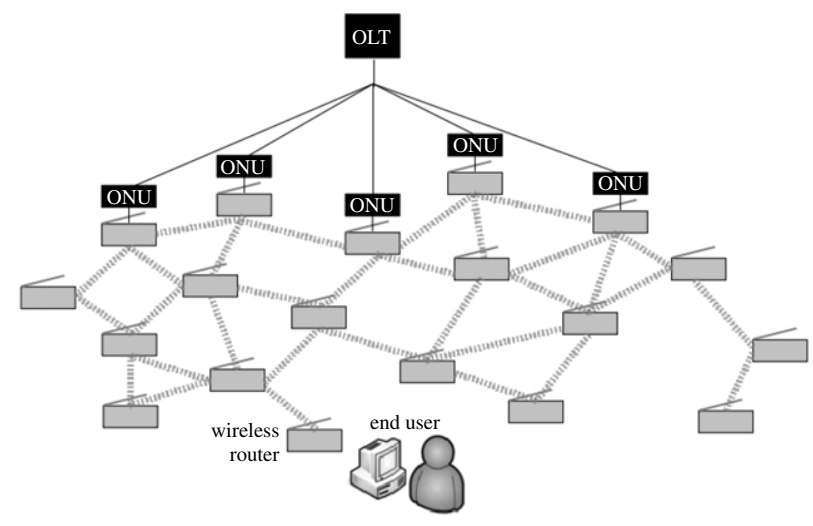

Fig. 1. A fiber-wireless access architecture.

The problem of switching optical or wireless devices to sleep mode is that energy saving and latency are competing objectives, as previously stated. Therefore, finding the optimal sleep mode scheduling system, which reduces energy consumption while keeping delay under a threshold, becomes a challenge. Here we address such a challenge in the context of FiWi access networks by developing a mathematical formalization and algorithm that may serve as a basis for planning and design of QoS-aware energy-efficient FiWi access networks.

The remainder of this paper is organized as follows. Section II discusses FiWi access architectures and their energy saving potential. In Section III the QoS-aware energy-efficient design problem under study is presented and formalized, and in Section IV an algorithmic approach to solve this problem is proposed. Section V analyzes simulations and results, while Section VI ends the article with some conclusions.

\section{ENERGy EFFiciency in FiWi ACCESs Networks}

\section{A. FiWi Access Architecture}

Although FTTx technologies are capable of offering veryhigh-performance access network connection to end users, these are still cost-prohibitive in many cases [8]. This fact has leveraged the emergence of hybrid wireless-optical technologies, where high-capacity optical back-end and wireless front-end are combined. This new access paradigm can provide very high throughputs in a cost-effective manner [9]. At the optical back-end a PON is usually used, because of low maintenance and high reliability, while WiFi and/or WiMax is used at the front-end. As illustrated in Fig. 1, PON segments coming out from the OLT, at the CO, connect to multiple ONUs using a tree configuration created by splicing fibers rather than using an electrical device. At the front-end, multiple wireless routers, usually having a mesh configuration, provide end-user access. The wireless routers directly connected to the ONUs are called gateways.

In FiWi access networks the end users gain network connectivity when connected to wireless routers. These wireless routers forward packets toward gateways where connectivity to the optical backhaul is provided. At the PON the ONUs contend for upstream bandwidth, using TDM, while the downstream traffic is broadcasted to all the ONUs. Each ONU will then discard any traffic not destined for it.

\section{B. The Energy Saving Potential of FiWi Access Architec- tures}

By joining wired and wireless technologies, FiWi access architectures not only become cost-effective solutions but also bring great opportunities for energy savings. Basically, this has to do with the impact on how traffic can be routed. Different routes, and different optical access points, can be adopted in order to allow some devices (wireless or optical) to switch to standby/sleep mode, without decreasing network utilization. However, such flexibility only becomes useful if the QoS experienced by users is kept at acceptable levels. More hops may lead to a decrease of the network throughput and an increase in delay. This is particularly true in broadcast media, such as the wireless section, because the medium is shared.

\section{State of the Art}

Techniques to reduce energy consumption have been applied to several network infrastructures. The studies in [10-12] focus on fixed wired IP networks, covering the backbone and metro sections. The model to estimate the energy consumption of the Internet in [11], besides the core and metro network sections, also considers the access network section. For PONs, sleep mode and adaptive link rate mechanisms, which switch the ONU mode and switch between downlink rates according to the amount of traffic, have been proposed [13,14]. Architectures for fast clock recovery have also been demonstrated enabling ONUs to have a fast transition from sleep to active mode [2,15]. In [2] different architectures are compared in terms of power consumption. The mechanism proposed in [16] considers a trade-off between maximizing the power saving and guaranteeing network performance in PONs.

Sleep mode approaches have also been widely exploited in wireless networks where battery consumption is of paramount importance [17]. The impact of QoS constraints on the energy efficiency of wireless transmissions has been analyzed in [18].

Energy efficiency in wireless-optical broadband access networks has been addressed in [4] where a mathematical formalization that acts as a guideline to the design of energyaware networks was presented. QoS-aware energy-efficient FiWi access networks have not been previously addressed, as far as we know.

\section{The QoS-AwARe EnERgY-EFFICIENT Design PROBLEM}

\section{A. Assumptions and Notation}

Let $\mathscr{O}$ be the set of all ONUs; $\mathscr{R}$ be the set of all wireless routers, gateways included; and $\mathscr{G}(\mathcal{V}, \mathscr{L})$ be the FiWi network graph, where $\mathcal{V}=\mathscr{O} \cup \mathscr{R} \cup$ OLT and $\mathscr{L}$ is the set of links. Let us also denote the set of sleep mode schedulings by $\mathscr{S}$. In general, 


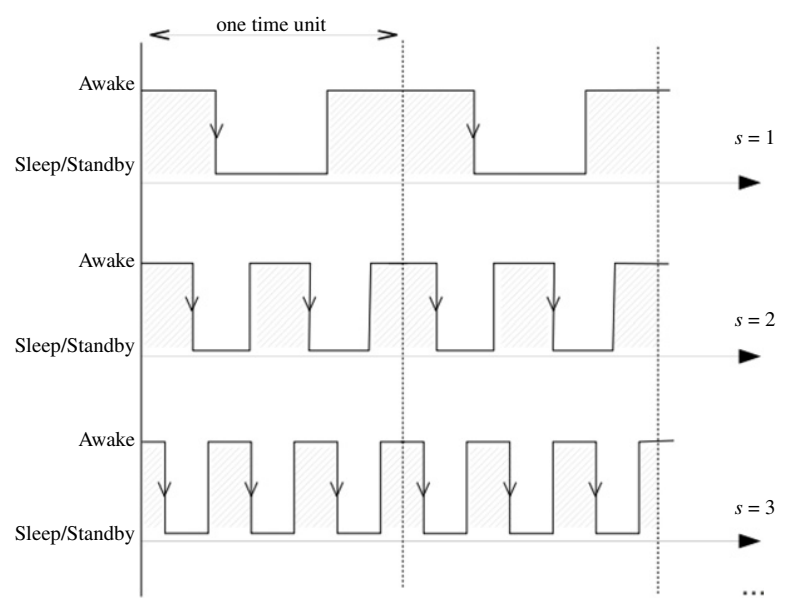

Fig. 2. The sleep mode scheduling scheme.

a scheduling $s \in \mathscr{S}$ defines how awake and sleep periods are arranged over time, and, obviously, any sleep mode scheduling system $\mathbf{s}=\left\{s_{v_{1}}, s_{v_{2}}, \ldots, s_{v_{n}}\right\}$, where $s_{v_{i}} \in \mathscr{S}, \forall v_{i} \in \mathcal{V} \backslash\{\mathrm{OLT}\}$, will induce the energy efficiency and delay at each $v_{i}$. The goal is to look for the optimal sleep mode scheduling system that maximizes energy efficiency while keeping network delay below a threshold, $\Delta^{T h}$, ensuring QoS.

In the adopted sleep mode scheduling scheme, a certain number of sleep periods per time unit is assigned to each ONU and wireless router. Such sleep periods will be interspersed with awake periods, as illustrated in Fig. 2. When looking into a single node, a small number of sleep periods entails longer sleep periods meaning that, since packets need to be queued when devices are in sleep mode, the more sleep periods are assigned to a device, the higher the QoS. This is so because such sleep periods will be of shorter duration. In terms of energy efficiency, spreading the total sleep time over many sleep periods entails more energy consumption because of the higher number of wake-ups. Choosing the right QoS-aware energy-efficient sleep mode scheduling system will depend on the load, delay threshold and energy consumptions of the operation modes. When a device never goes to sleep, then $s_{v}=0, v \in \mathscr{V} \backslash\{$ OLT $\}$. If $s_{v}=s, s \in \mathscr{S}$, then $s_{v}$ will have $s$ sleep periods per time unit.

In the following sections, the downstream and upstream traffic to/from $r \in \mathscr{R}$ are denoted by $D_{r}$ and $U_{r}$, respectively, while $C_{\mathscr{R}}$ and $C_{\mathscr{O}}$ are the transfer capacities of the wireless routers and ONUs, respectively. All of them are assumed to be in packets per second. The average packet size in bytes is denoted by $P$.

To evaluate the energy efficiency of sleep mode schedulings the following energy consumption values become necessary. These are one-byte-time-related values, where one byte time refers to the time that one byte takes to be transmitted. That is, the following energy values are average energy consumptions during one byte time:

$E_{\mathscr{O}}^{\text {Wake }}$ Energy consumed by ONUs in awake mode.

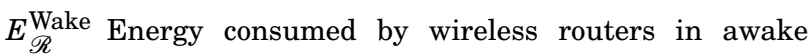
mode. $\begin{aligned} E_{\mathscr{O}}^{\text {Sleep }} & \text { Energy consumed by ONUs in sleep mode. } \\ E_{\mathscr{R}}^{\text {Standby }} & \begin{array}{l}\text { Energy consumed by wireless routers in standby } \\ \text { mode. }\end{array}\end{aligned}$

When evaluating the overall energy efficiency, the durations of the awake, standby/sleep and wake-up periods, together with the number of transitions from standby/sleep to awake mode, must be accounted for. In the following discussion this will be done considering a time window of one second. The notation related to the energy cost and delay, induced by the scheduling system $\mathbf{s}$, will be as follows:

$\rho_{v}(\mathbf{s})$ Total energy cost at $v \in \mathcal{V} \backslash\{\mathrm{OLT}\}$.

$\Delta_{v}^{s}(\mathbf{s})$ Average packet delay if scheduling $s \in \mathscr{S}$ is used at node $v \in \mathcal{V} \backslash\{\mathrm{OLT}\}$.

$\omega_{v}(\mathbf{s})$ Wake-up energy cost at $v \in \mathcal{V} \backslash\{\mathrm{OLT}\}$.

The variables are as follows:

$\mathbf{s}$ Sleep mode scheduling system, where $\mathbf{s}=\left\{s_{v_{1}}, s_{v_{2}}\right.$, $\left.\ldots, s_{v_{n}}\right\}, \forall s_{v_{i}} \in \mathscr{S}, \forall v \in V \backslash\{\mathrm{OLT}\}$.

$\delta_{r, l}$ Flow allocation on link $l \in \mathscr{L}$ for downstream traffic from the OLT toward $r \in \mathscr{R}$.

$\mu_{r, l}$ Flow allocation on link $l \in \mathscr{L}$ for upstream traffic from $r \in \mathscr{R}$ toward the OLT.

$\gamma_{v}$ Load at node $v \in \mathcal{V} \backslash\{\mathrm{OLT}\}$.

\section{B. Problem Formalization}

- Objective Function: To improve energy efficiency, the total energy cost of both optical and wireless sections of the FiWi must be minimized using

$$
\text { Minimize }_{\mathbf{s}} \sum_{v \in \mathcal{V} \backslash\{\mathrm{OLT}\}} \rho_{v}(\mathbf{s}) .
$$

The constraints to the problem being formalized are discussed next. Please note that the downstream and upstream traffic are not conditioned to take a particular route. That is, traffic emanating from a source node will reach the optical section using the links that best serve the purpose of improving energy efficiency. In this way, the best scenario in terms of energy efficiency is being considered. According to the assigned flow allocation, the traffic load of every node is determined and used in delay- and energy-efficiency-related constraints.

- Flow Conservation: Downstream and upstream traffic between wireless routers and the OLT are subject to the following flow conservation law:

$$
\begin{gathered}
\sum_{l \in \mathscr{L}: s(l)=v} \delta_{r, l}-\sum_{l \in \mathscr{L}: d(l)=v} \delta_{r, l}= \begin{cases}D_{r}, & \text { if } v=\mathrm{OLT}, \\
-D_{r}, & \text { if } v=r, \\
0, & \text { otherwise, }\end{cases} \\
\forall r \in \mathscr{R}, \forall v \in \mathcal{V}, \\
\sum_{l \in \mathscr{L}: s(l)=v} \mu_{r, l}-\sum_{l \in \mathscr{L}: d(l)=v} \mu_{r, l}=\left\{\begin{aligned}
U_{r}, & \text { if } v=r, \\
-U_{r}, & \text { if } v=\text { OLT, } \\
0, & \text { otherwise, }
\end{aligned}\right. \\
\forall r \in \mathscr{R}, \forall v \in \mathcal{V},
\end{gathered}
$$


where $s(l)$ and $d(l)$ are the source and destination nodes of link $l \in \mathscr{L}$, respectively.

- Load Factor: The load factor expressed in this section is not the number of packets per second passing through a device. Instead, this is the load value that will determine when the circuitry can go to sleep and will influence delay. For the ONUs it is assumed that the OLT controller matches the downstream traffic slots with the allocated upstream traffic slots, as proposed in [15]. Thus, for each ONU, the highest value between its upstream and downstream traffic will become its load factor:

$$
\gamma_{o}=\max \left(\sum_{l \in \mathscr{L}: s(l)=o} \sum_{r \in \mathscr{R}} \delta_{r, l}, \sum_{l \in \mathscr{L}: s(l)=o} \sum_{r \in \mathscr{R}} \mu_{r, l}\right), \quad \forall o \in \mathscr{O} .
$$

Since the medium of transmission in wireless networks is broadcast, the load factor that determines when the circuitry of a wireless router can go to standby mode, and will influence delay, is given by

$$
\gamma_{r}=\sum_{l \in \mathscr{L}: s(l)=r \vee d(l)=r} \sum_{r \in \mathscr{R}}\left(\mu_{r, l}+\delta_{r, l}\right), \quad \forall r \in \mathscr{R} .
$$

- Limitation of Delay: To provide QoS guarantees, the average packet delay will be kept below the threshold $\Delta^{T h}$,

$$
\sum_{v \in \mathcal{V} \backslash\{\mathrm{OLT}\}} \Delta_{v}^{s}(\mathbf{s}) \leq \Delta^{T h}, \quad \forall s \in \mathscr{S}
$$

Assuming that the fraction of time that a wireless router or ONU $v$ is awake is given by $\frac{\gamma_{v}}{C_{v}}$, where $C_{v}=C_{\mathscr{R}}$ or $C_{v}=C_{\mathscr{O}}$ according to whether it refers to a wireless router or ONU, and assuming a Poisson model for traffic arrivals, the average packet delay when scheduling $s \in \mathscr{S}$ is used at node $v \in \mathcal{V}$, denoted by $\Delta_{v}^{S}(\mathbf{s})$, can be approximated by

$$
\Delta_{v}^{s}(\mathbf{s})=\left\{\begin{array}{cl}
\frac{1}{C_{v}-\gamma_{v}}, & \text { if } s=0, \\
\frac{1}{C_{v}-\gamma_{v}}+\frac{\gamma_{v}}{C_{v}} \frac{C_{v}-\gamma_{v}}{C_{v}} \frac{1}{s}, & \text { otherwise, } \\
\forall v \in \mathcal{V} \backslash\{\mathrm{OLT}\}, &
\end{array}\right.
$$

assuming $C_{v}>\gamma_{v}$ (otherwise, packet dropping is assumed). For $s=0$ (always awake mode) the delay will be the average packet delay associated with Poisson arrivals, $\frac{1}{C_{v}-\gamma_{v}}$, from queueing theory. For $s \neq 0$ one has to consider that packets may arrive during awake and sleep periods. When devices are in standby/sleep, besides the average packet delay for Poisson arrivals, a delay associated with the fact that packets will be queued, and transmitted after the wake-up process, must be added. This is captured by $\frac{\gamma_{v}}{C_{v}} \frac{C_{v}-\gamma_{v}}{C_{v}} \frac{1}{s}$, where the transmission delay associated with all the load at node $v, \frac{\gamma_{v}}{C_{v}}$, is multiplied by the fraction of time that the node is in sleep mode, $\frac{C_{v}-\gamma_{v}}{C_{v}}$, in order to capture only the fraction of packets arriving during sleeping. The final $\frac{1}{s}$ reflects the fact that more sleep periods (and, therefore, shorter sleep periods) lead to fewer packets accumulated in queues.

Please note that, according to this formalization, sleep periods may follow the dynamic growth of queues as long as the number of sleep periods and total sleep time does not change for a specific scheduling $s$. That is, the sleep periods in Fig. 2 may shrink or grow, according to a queue size threshold, for example, as long as the number of sleep periods and total sleep time do not change for the scheduling $s$ being considered.

- Energy Cost: To evaluate the energy cost per wireless router and ONU, the durations of the awake, standby/sleep and wake-up periods, together with the number of transitions from standby/sleep to awake mode, must be considered. Assuming that the wake-up energy costs for wireless routers and ONUs are given by

$$
\begin{aligned}
& \omega_{r}(\mathbf{s})=s_{r} \times E_{\mathscr{R}}^{\text {Wake }} \times W_{\mathscr{R}}, \\
& \omega_{O}(\mathbf{s})=s_{O} \times E_{\mathscr{O}}^{\text {Wake }} \times W_{\mathscr{O}},
\end{aligned}
$$

where $W_{\mathscr{R}}$ and $W_{\mathscr{O}}$ are the numbers of byte times that the wake-up process takes for wireless routers and ONUs, respectively, then the energy costs per wireless router and ONU during one second will be

$$
\begin{gathered}
\rho_{r}(\mathbf{s})=\left\{\begin{array}{cl}
C_{\mathscr{R}} \times P \times E_{\mathscr{R}}^{\mathrm{Wake},} & \text { if } s_{r}=0, \\
\gamma_{r} \times P \times E_{\mathscr{R}}^{\mathrm{Wake}}+\omega_{r}(\mathbf{s}) & \text { or } \gamma_{r} \geq C_{\mathscr{R}}, \\
+P\left(C_{\mathscr{R}}-\gamma_{r}-\frac{s_{r} \times W_{\mathscr{R}}}{P}\right) E_{\mathscr{R}}^{\mathrm{Standby}}, & \text { otherwise }
\end{array}\right. \\
\rho_{o}(\mathbf{s})=\left\{\begin{array}{cl}
C_{\mathscr{O}} \times P \times E_{\mathscr{O}}^{\mathrm{Wake}}, & \text { if } s_{o}=0, \\
\gamma_{o} \times P \times E_{\mathscr{O}}^{\mathrm{Wake}}+\omega_{o}(\mathbf{s}) & \text { or } \gamma_{o} \geq C_{\mathscr{O}}, \\
+P\left(C_{\mathscr{O}}-\gamma_{o}-\frac{s_{o} \times W_{\mathscr{O}}}{P}\right) E_{\mathscr{O}}^{\mathrm{Sleep}}, & \text { otherwise } \\
\forall o \in \mathscr{O} .
\end{array}\right.
\end{gathered}
$$

- Non-Negative Assignments:

$$
\mathbf{s}, \gamma_{v}, \delta_{r, l}, \mu_{r, l} \geq 0 \text {. }
$$

\section{Hardness of the Problem}

When expanding expressions (1) and (6) to uncompact notation we end up with

$$
\begin{gathered}
\text { Minimize }_{\mathbf{s}} \sum_{v \in \mathcal{V} \backslash \mathrm{OLT}} \rho_{v, s} x_{v, s}, \\
\sum_{s \in \mathscr{S}} x_{v, s}=1, \quad \forall v \in \mathcal{V} \backslash\{\mathrm{OLT}\}, \\
\sum_{v \in \mathcal{V} \backslash\{\mathrm{OLT}\}} \Delta_{v, s} x_{v, s} \leq \Delta^{T h}, \quad \forall s \in \mathscr{S},
\end{gathered}
$$

where $x_{v, s} \in\{0,1\}$ indicates whether node $v$ is using scheduling $s$ or not, $\rho_{v, s}$ is the energy cost of $v$ when using scheduling $s$ and $\Delta_{v, s}$ is the packet delay of $v$ when using scheduling $s$. This happens to be a generalized assignment problem (GAP) with non-linear costs and non-linear resource constraints. The GAP seeks a minimum cost assignment of $n$ jobs to $m$ agents, where one job is assigned to exactly one agent, while satisfying a resource constraint for each agent. In our case, the wireless 


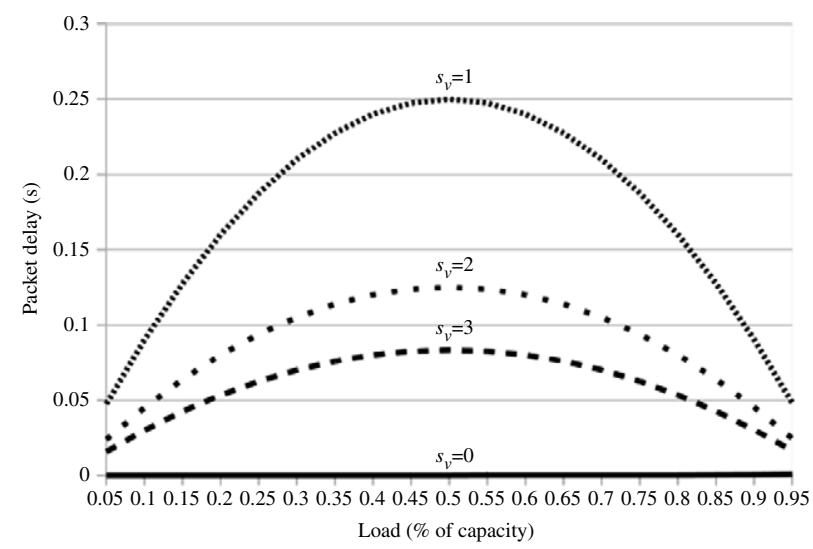

Fig. 3. Plot of packet delay assuming a router capacity of $100 \mathrm{Mbps}$.

routers and ONUs can be seen as the jobs, the sleep mode schedulings can be seen as the agents, the average delays at wireless routers and ONUs can be seen as the resources used by jobs and a delay threshold is a resource constraint for an agent. The problem being addressed here includes, therefore, a flow problem and a GAP. This fact underlies the strategy proposed next to solve it.

The GAP is known to be NP-hard [19] and the problem of judging the existence of a feasible solution for a GAP is already known to be NP-complete [20]. Thus, the QoS-aware energy-efficient design problem is NP-hard as well.

\section{Algorithmic Approach for the QoS-AwARE EnERgy-EFficient Design Problem}

\section{A. Problem Characteristics}

We state next two claims that provide the foundation for the structure of the proposed algorithm. These will be adopted when assigning sleep mode schedulings to nodes while satisfying the delay constraint.

1) Delay Curves: When plotting expression (7) for different sleep mode schedulings, shown in Fig. 3, we observe that when there are sleep periods $s_{v}>0$, the smaller the number of sleep periods (low $s_{v}$ value) the larger the delay increase, except for very low or very high loads. The fact that a small number of sleep periods also induces less energy cost (see expressions (10) and (11)) leads us to the next assumption.

Claim 1. A good solution will assign a small number of sleep periods to nodes having very low or very high (near the capacity) loads. For moderate loads, more sleep periods should be assigned since the average packet delay increase tends to smooth.

2) Sleep Condition: When accounting for the energy consumption in a wireless node or ONU device, it is clear that such a device should only go to standby/sleep when the energy consumption in the awake mode is higher than the corresponding energy consumption in the standby/sleep mode and wake-up-related energy consumption together. A wireless router $r \in \mathscr{R}$, for example, benefits from scheduling $s_{r}$ if

$$
\begin{gathered}
C_{\mathscr{R}} \times P \times E_{\mathscr{R}}^{\text {Wake }}>\gamma_{r} \times P \times E_{\mathscr{R}}^{\text {Wake }}+\omega_{r}\left(s_{r}\right) \\
+\left(C_{\mathscr{R}}-\gamma_{r}-\frac{s_{r} \times W_{\mathscr{R}}}{P}\right) \times P \times E_{\mathscr{R}}^{\text {Standby }} \\
\Leftrightarrow \gamma_{r}<C_{\mathscr{R}}-\frac{s_{r} \times W_{\mathscr{R}}}{P} .
\end{gathered}
$$

A similar condition for an $\mathrm{ONU} o \in \mathscr{O}$ will be

$$
\begin{gathered}
C_{\mathscr{O}} \times P \times E_{\mathscr{O}}^{\text {Wake }}>\gamma_{o} \times P \times E_{\mathscr{O}}^{\text {Wake }}+\omega_{o}\left(s_{r}\right) \\
+\left(C_{\mathscr{O}}-\gamma_{o}-\frac{s_{o} \times W_{\mathscr{O}}}{P}\right) \times P \times E_{\mathscr{O}}^{\text {Sleep }} \\
\Leftrightarrow \gamma_{o}<C_{\mathscr{O}}-\frac{s_{o} \times W_{\mathscr{O}}}{P} .
\end{gathered}
$$

Therefore, we can make the following assumption.

Claim 2. No sleep mode scheduling should be assigned to a routerr $\left(s_{r}=0\right)$ when condition (16) is violated. Similarly, no sleep mode scheduling should be assigned to an ONUo $\left(s_{o}=0\right)$ when condition (17) is violated.

\section{B. Two-Step Approach}

The QoS-aware energy-efficient design problem being addressed includes a flow problem and a GAP that are linked by packet delay constraints. This fact suggests to us a two-step approach that would use the flow variable values, obtained when solving the flow subproblem independently, to feed the GAP subproblem. More specifically, the two steps of such approach would be the following:

Step 1 Determine good approximation values for the flowrelated variables $\delta_{r, l}, \mu_{r, l}$ and $\gamma_{v}$ considering no sleep mode scheduling assignment in particular. These variables specify how traffic from source nodes will be delivered to the optical section. Reduce the QoS-aware energy-efficient design problem to a non-linear GAP by fixing these flow-related variables.

Step 2 Heuristically solve the resulting non-linear GAP having the previously stated claims as a basis for sleep mode scheduling assignment, while satisfying the packet delay constraint.

1) Step 1: It is reasonable to assume that the best solutions to our problem will end up having few hops for downstream and upstream traffic flow while making an even traffic distribution among nodes. This is so because more hops mean more delay and waste of energy. Thus, traffic congestion should be minimized while keeping the number of hops small, which can be achieved by the next LP problem:

$$
\begin{gathered}
\text { Minimize: } T_{\mathscr{R}}+\alpha \times T_{\mathscr{O}}, \\
\text { Subject to: } \gamma_{r} \leq T_{\mathscr{R}}, \quad \forall r \in \mathscr{R}, \\
\gamma_{o} \leq T_{\mathscr{O}}, \quad \forall o \in \mathscr{O},
\end{gathered}
$$




$$
\begin{aligned}
\sum_{r \in \mathscr{R}} \sum_{l \in \mathscr{L}} \frac{\delta_{r, l}}{D_{r}} & \leq H \times|\mathscr{R}|, \\
\sum_{r \in \mathscr{R}} \sum_{l \in \mathscr{L}} \frac{\mu_{r, l}}{U_{r}} & \leq H \times|\mathscr{R}|, \\
T_{\mathscr{R}}, T_{\mathscr{O}} & \geq 0,
\end{aligned}
$$

plus conditions (2)-(5) and (12), where $H$ is the average number of hops, for network traffic flow, when using the shortest path. The $\alpha$ is a value small enough so that minimizing the traffic congestion threshold $T_{\mathscr{R}}$ is preferred to minimizing the traffic congestion threshold $T_{\mathscr{O}}$, as there are more wireless nodes than ONUs.

2) Step 2: Using $\gamma_{v}$ obtained in Step 1 as input, determine a feasible solution for $\mathbf{s}$ as follows:

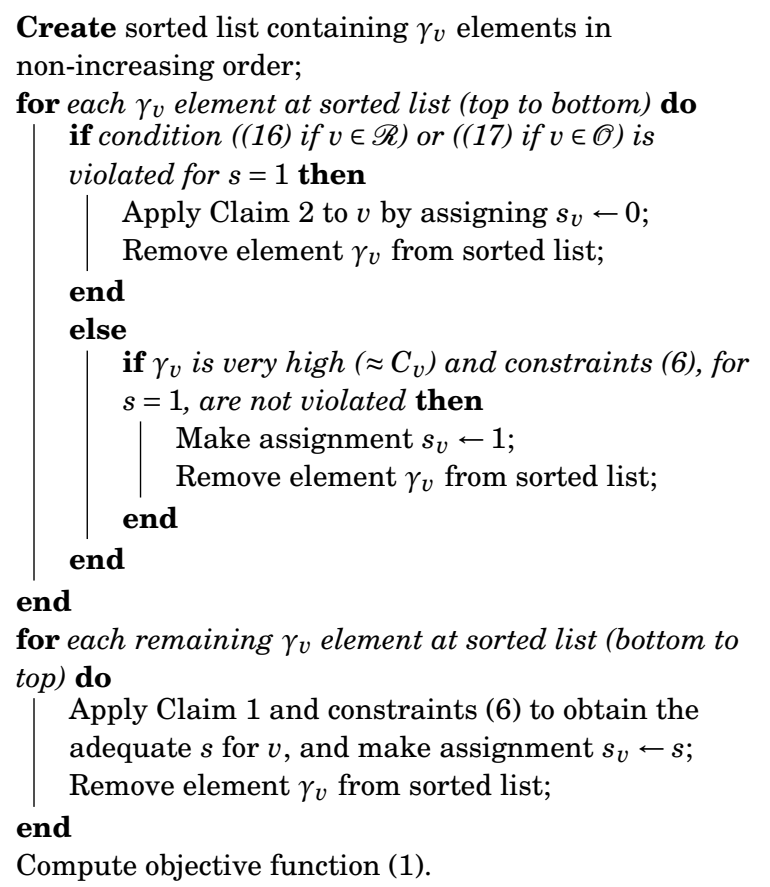

According to rows 4-8, no sleep mode scheduling is assigned to a node $v$ if condition (16) or (17), depending on whether $v$ is a router or an ONU, is violated for $s=1$ (if this condition is violated for $s=1$, the less energy-consuming sleep mode scheduling, then it is also violated for $s>1$ ). That is, the node will not benefit from any sleep mode scheduling. Rows 10-14 assign the sleep mode scheduling $s=1$ to heavily loaded nodes if the corresponding delay constraint in expression (6) is not violated. For the remaining nodes, rows 17-22 will find the best sleep mode scheduling according to claim 1 and constraints (6).

\section{Analysis of Results}

In this section we will analyze the energy cost and average packet delay behavior when using the proposed approach to design QoS-aware energy-efficient FiWi access networks. The results will be compared with a lower and an upper bound discussed next.

\section{A. Upper and Lower Bounds}

It is clear that the problem being addressed has two conflicting goals: minimization of energy consumption and minimization of global network packet delay. In terms of network energy cost, we can therefore define a lower bound (LB) and an upper bound (UB) as follows:

$\rho^{\mathrm{LB}}$ - Solve a linear problem using objective function (1), while setting $s_{v}=1, \forall v \in \mathcal{V}$, and constraints from (2) to (5). This linear problem does not take packet delay into account, optimizing energy cost only. The network packet delay associated with $\rho^{\mathrm{LB}}$ can be obtained by adding the average packet delay (expression (7)) of all nodes, while setting $s=1$ and using the network loads resulting from $\rho^{\mathrm{LB}}$ (that is, using the $\gamma_{v}$ values resulting from the $\rho^{\mathrm{LB}}$ solution).

$\rho^{\mathrm{UB}}-$ Due to the energy cost and packet delay conflict, the worst network energy cost (and, therefore, best network packet delay) can be obtained from expressions (10) and (11) while setting $s_{r}=0$ and $s_{o}=0$, respectively. This is an upper bound for any load scenario, depending only on the size of the network. In this case the packet delay at each node is approximately 0 , as can be seen in Fig. 3, meaning that the total network packet delay can also be considered to be 0 .

These bounds will be used to analyze the energy cost and average packet delay in the following sections.

\section{B. Energy Consumption}

Assuming that the ONUs use $3.85 \mathrm{~W}$ in awake mode, $750 \mathrm{~mW}$ in sleep mode and take $3.5 \mathrm{~ms}$ to wake up, while the wireless routers consume $1.75 \mathrm{~W}$ in transmit mode, $0.75 \mathrm{~W}$ in receive mode, $10 \mathrm{~mW}$ in standby mode and take $5 \mathrm{~ms}$ to wake up, according to [2,21], we obtain the following energy (Joules) consumption values during one byte time:

$$
\begin{aligned}
& E_{\mathscr{O}}^{\text {Wake }}=30.8 \times 10^{-9} \mathrm{~J}, \\
& E_{\mathscr{R}}^{\text {Wake }}=1.0 \times 10^{-7} \mathrm{~J}, \\
& E_{\mathscr{O}}^{\text {Sleep }}=6.0 \times 10^{-9} \mathrm{~J}, \\
& E_{\mathscr{R}}^{\text {Standby }}=8.0 \times 10^{-10} \mathrm{~J} .
\end{aligned}
$$

These are the energy consumption values adopted in our simulations. For $E_{\mathscr{R}}^{\text {Wake }}$ the average of the energies consumed when in transmit and receive modes is assumed. The values for $W_{\mathscr{O}}$ and $W_{\mathscr{R}}$ will be $\frac{3.5 \mathrm{~ms}}{\frac{1}{C_{\mathscr{O}} \times P}}=437,500$ and $\frac{5 \mathrm{~ms}}{\frac{1}{C_{\mathscr{R}} \times P}}=$ 62,500 , respectively. It is assumed that the ONUs and the wireless routers have capacities of $1 \mathrm{Gbps}$ and $100 \mathrm{Mbps}$, respectively, meaning that $C_{\mathscr{O}}=250,000$ and $C_{\mathscr{R}}=25,000$ packets, considering a packet size of 500 bytes $(P=500)$.

The byte times for the wireless routers and ONUs are

$$
\begin{aligned}
\text { Byte time for wireless routers } & =\frac{1}{C_{\mathscr{R}} \times P} \mathrm{~s}, \\
\text { Byte time for ONUs } & =\frac{1}{C_{\mathscr{O}} \times P} \mathrm{~s} .
\end{aligned}
$$



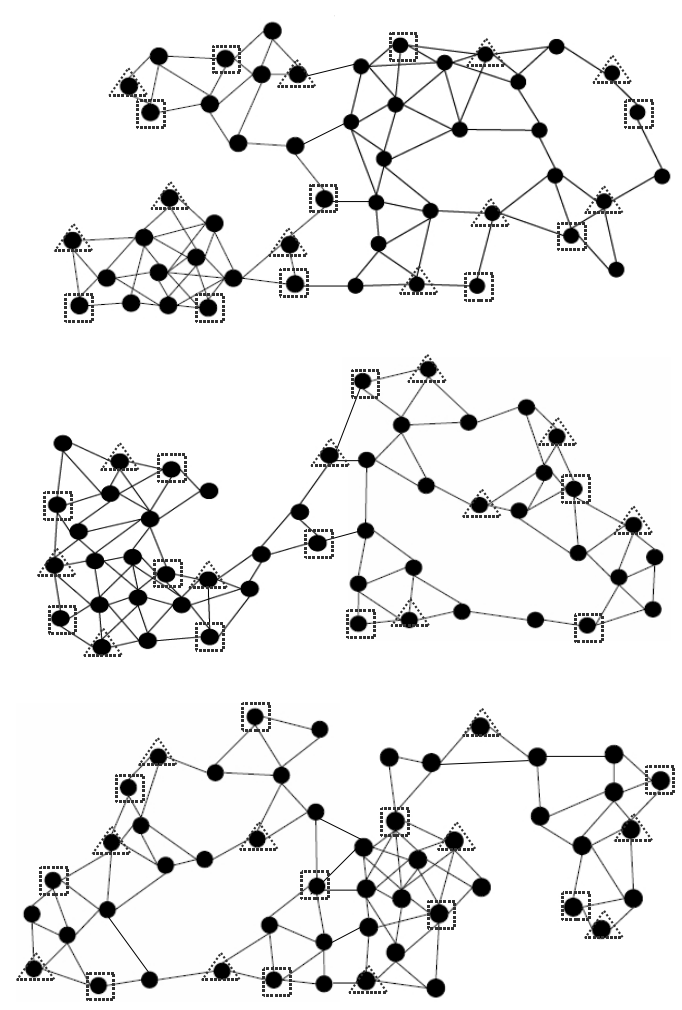

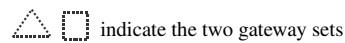

Fig. 4. The randomly generated networks used in the simulations.

\section{Test Data}

Simulations were carried out for three randomly generated networks of 50 wireless routers each, shown in Fig. 4, using the weighted proximity algorithm presented in [22]. For the gateways, two sets of ten nodes, randomly selected using a uniform distribution, were considered for each network. For each of these six scenarios, simulations were carried out for traffic demands (both upstream and downstream traffic demands at each wireless router) from 0 to $10 \%, 20 \%$ and $30 \%$ of $C_{\mathscr{R}}$, randomly generated using a uniform distribution. Such upstream and downstream traffic will traverse the necessary wireless routers to reach the optical section, meaning that it will contribute to the load of more than one node. In our scenarios, an ONU serves one gateway.

The two steps of the algorithm, presented in Subsection IV.B, were solved using the CPLEX optimization package and a programming routine in $\mathrm{C}$, respectively. The algorithm assumes that $\mathscr{S}=\{0,1,2,3,4\}$, for both wireless routers and ONUs, which means at most 4 sleep periods may exist per time unit. The running times, using a $3.0 \mathrm{GHz}$ Pentium processor with 1 GB RAM, for both steps were lower than one second, meaning that this algorithmic approach is computationally efficient.

The plots discussed next present average, minimum and maximum energy cost and packet delay values, obtained from all randomly generated networks (sets of gateways included), for different node demands. The delay thresholds $\Delta^{T h}$ tested

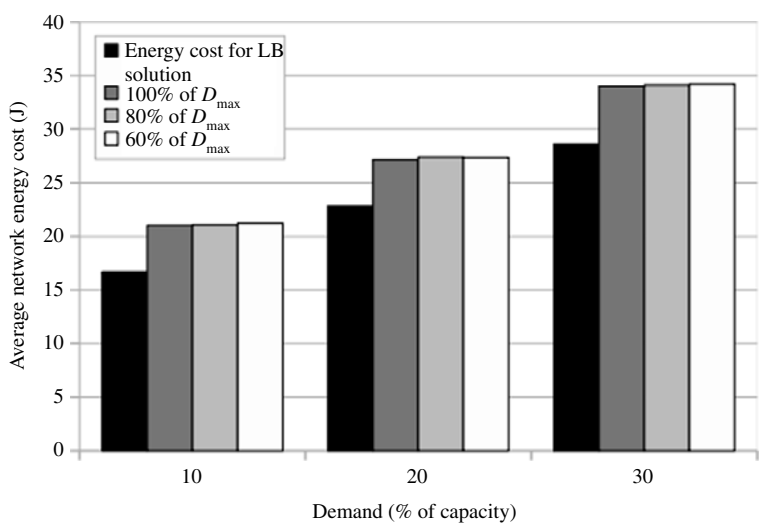

Fig. 5. Average network energy costs.

were $100 \% \times D_{\max }, 80 \% \times D_{\max }$ and $60 \% \times D_{\max }$, where

$$
D_{\max }=\frac{\sum_{v \in \mathcal{M} \backslash\{\text { OLT }\}} \frac{1}{C_{v}-\gamma_{v}}+\frac{\gamma_{v}}{C_{v}} \times \frac{C_{v}-\gamma_{v}}{C_{v}}}{|\mathscr{S}|} .
$$

The upper part is basically the largest possible network delay,

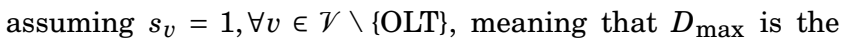
largest per scheduling mode delay. The plotted energy cost and packet delay values are both global-network-related values (all per-node values are added).

\section{Energy Efficiency and Average Packet Delay Analysis}

The plot in Fig. 5 shows the average network energy cost values obtained by the proposed two-step heuristic approach, for different node demands and different delay thresholds, and the values obtained by the lower bound on network energy cost, $\rho^{\mathrm{LB}}$. From this plot it is possible to conclude that our approach allows a reduction of the average packet delay, through delay threshold reduction, with almost no increase in the network energy cost, down to $\Delta^{T h}=60 \% \times D_{\max }$. This can be confirmed by Table I where the cumulative frequencies of sleep mode schedulings involved in the solutions are exhibited. These are average values taken from the solutions obtained for the six scenarios that were tested. As it is possible to see, nodes were always able to go to sleep mode. For tighter $\Delta^{T h}$ values (less than $60 \% \times D_{\max }$ ) the energy cost increases significantly, meaning that the obtained solutions are not acceptable from the energy point of view. ${ }^{1}$ The difference between the values obtained by $\rho^{\mathrm{LB}}$ and the values obtained by the heuristic is maintained for different node demands, which means that this approach is scalable.

The plot in Fig. 6 shows the average network packet delays obtained by the proposed two-step heuristic approach, for different node demands and different delay thresholds, and the network packet delay associated with $\rho^{\mathrm{LB}}$. This plot confirms that the average packet delay decreases as the delay threshold, $\Delta^{T h}$, becomes tighter. For $\Delta^{T h}<60 \% \times D_{\max }$, more nodes need to be in awake mode, in order to satisfy the very tight delay constraints, which leads to a significant increase in energy cost.

1 These results were intentionally not included in the plots because they would disturb the display of relevant values. 
TABLE I

Average Cumulative Frequencies of Sleep Mode Schedulings InVOLVED IN THE Solutions (\%).

\begin{tabular}{lcccc}
\hline & & \multicolumn{3}{c}{$\Delta^{T h}$} \\
\cline { 3 - 5 } & & $100 \% \times$ & $80 \% \times$ & $60 \% \times$ \\
& & $D_{\max }$ & $D_{\max }$ & $D_{\max }$ \\
\hline \multirow{2}{*}{ Demand of } & $s=0$ & 0 & 0 & 0 \\
$10 \%$ & $s \leq 2$ & 30 & 26 & 20 \\
& $s \leq 3$ & 100 & 58 & 47 \\
& $s \leq 4$ & 100 & 92 & 76 \\
Demand of & $s=0$ & 0 & 0 & 100 \\
$20 \%$ & $s \leq 1$ & 28 & 24 & 0 \\
& $s \leq 2$ & 63 & 53 & 44 \\
& $s \leq 3$ & 100 & 90 & 73 \\
& $s \leq 4$ & 100 & 100 & 100 \\
Demand of & $s \leq 1$ & 0 & 0 & 0 \\
$30 \%$ & $s \leq 2$ & 59 & 22 & 17 \\
& $s \leq 3$ & 100 & 49 & 39 \\
& $s \leq 4$ & 100 & 100 & 67 \\
\hline
\end{tabular}

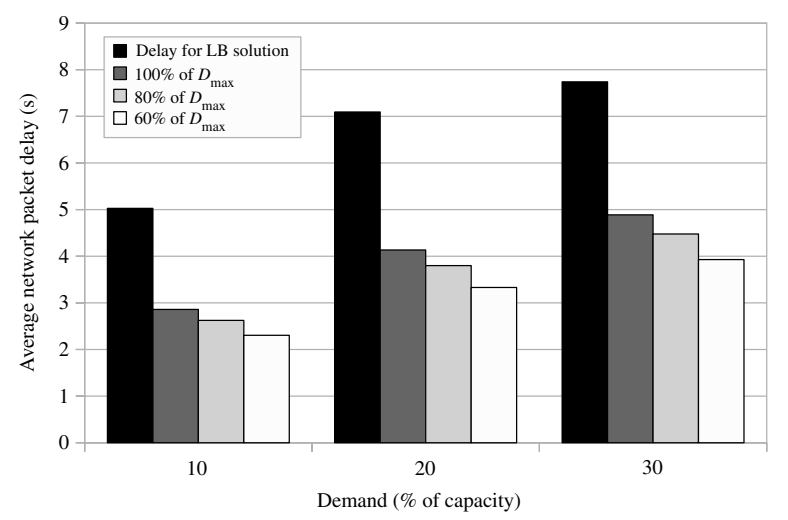

Fig. 6. Average network packet delays.

When compared with the packet delay associated with $\rho^{\mathrm{LB}}$, the energy cost lower bound solution, we can observe that the delay reduction is very significant $(\approx 50 \%$ or $\approx 40 \%)$. This is a very good achievement since this is obtained at the expense of almost no increase in energy cost. Please note that these are global network delays. The average delay per node will be around 50, 70 and $90 \mathrm{~ms}$ for node demands of $10 \%, 20 \%$ and $30 \%$, respectively.

Table II shows the average, minimum and maximum network energy costs obtained by the proposed two-step approach. We can say that the algorithm is stable, presenting a consistent behavior, as the difference is maintained when the demand increases. This can be confirmed from Fig. 7. A similar observation can be made concerning the network packet delay values obtained by the proposed two-step approach, as shown in Table III and Fig. 8. The delay values relate to the entire network (all per node delay values were added).

The worst network energy cost, $\rho^{\mathrm{UB}}$, obtained from expressions (10) and (11) while setting $s_{r}=0$ and $s_{O}=0$, respectively, will be $88.5 \mathrm{~J}$ for the 50 node networks used in our simulations, also considering a time window of one second. The significant difference between this value and the ones shown
TABLE II

Average, Minimum and Maximum Network Energy Costs Obtained By THE Two-STEP HeURISTIC APPROACH (JOULES).

\begin{tabular}{lllll}
\hline & & \multicolumn{3}{c}{$\Delta^{T h}$} \\
\cline { 3 - 5 } & & $100 \% \times$ & $80 \% \times$ & $60 \% \times$ \\
& & $D_{\max }$ & $D_{\max }$ & $D_{\max }$ \\
\hline \multirow{2}{*}{ Demand of } & Avg & 21.008 & 21.083 & 21.215 \\
& Min & 20.373 & 20.481 & 20.564 \\
& Max & 21.516 & 21.619 & 21.732 \\
\multirow{2}{*}{ Demand of } & Avg & 27.164 & 27.404 & 27.369 \\
& Min & 25.792 & 25.894 & 25.954 \\
\multirow{2}{*}{ Demand of } & Max & 28.421 & 28.545 & 28.616 \\
$30 \%$ & Avg & 33.982 & 34.095 & 34.185 \\
& Min & 32.628 & 32.725 & 32.914 \\
\hline
\end{tabular}

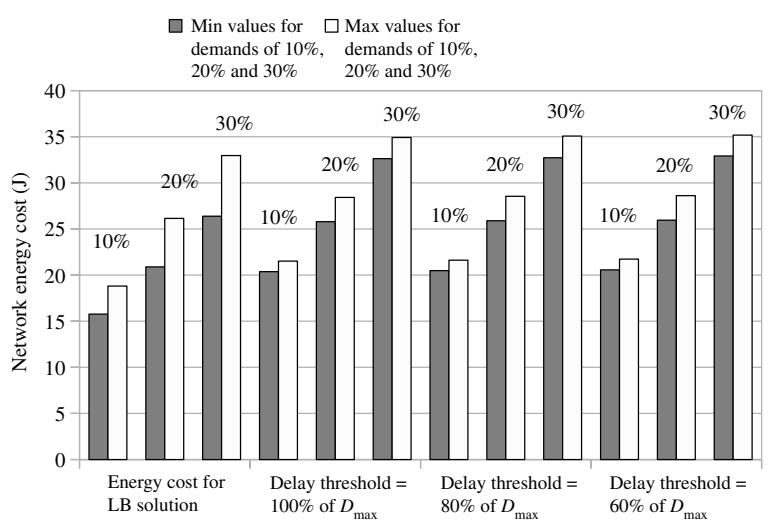

Fig. 7. Minimum and maximum network energy costs among all topology and gateway scenarios generated.

in Table II (between 20 and $35 \mathrm{~J}$ ) reveals that huge energy cost savings (from $60 \%$ to $77 \%$ ) can be obtained by adequately putting to sleep and waking up devices. Moreover, these energy savings are possible while keeping the increase in delay restricted (less than $90 \mathrm{~ms}$ per node, per second). Nowadays, energy saving has become one of the most critical issues in access networks, where the demand for digital traffic, and consequently energy cost, continues to grow. Approaches such as the one proposed here can, therefore, strongly contribute to making these networks much more energy-efficient.

As a final conclusion we can state that the two-step approach proposed here, besides being computationally efficient and stable, is able to find energy-efficient sleep mode scheduling systems while keeping delay under a threshold. This approach may, therefore, serve as a basis for planning and design of QoS-aware energy-efficient FiWi access networks.

\section{CONCLUSIONS}

In this article we have developed a mathematical formalization and an algorithm to find the proper way of putting to sleep and waking up devices in FiWi access networks, so that energy consumption is reduced and packet delay is kept under a threshold. The results show that the proposed approach is 
TABLE III

Average, Minimum and Maximum Network Packet Delays ObTained by the Two-Step Heuristic APPROACH (SECONDS).

\begin{tabular}{lllll}
\hline & & \multicolumn{3}{c}{$\Delta^{T h}$} \\
\cline { 3 - 5 } & & $100 \% \times$ & $80 \% \times$ & $60 \% \times$ \\
& & $D_{\max }$ & $D_{\max }$ & $D_{\max }$ \\
\hline \multirow{2}{*}{ Demand of } & Avg & 2.859 & 2.623 & 2.304 \\
& Min & 2.746 & 2.511 & 2.204 \\
& Max & 2.946 & 2.709 & 2.39 \\
\hline \multirow{2}{*}{ Demand of } & Avg & 4.134 & 3.798 & 3.329 \\
& Min & 3.852 & 3.56 & 3.113 \\
\hline \multirow{2}{*}{ Demand of } & Max & 4.377 & 4.025 & 3.508 \\
& Avg & 4.887 & 4.476 & 3.928 \\
& Min & 4.353 & 4.01 & 3.51 \\
& Max & 5.028 & 4.57 & 4.01 \\
\hline
\end{tabular}

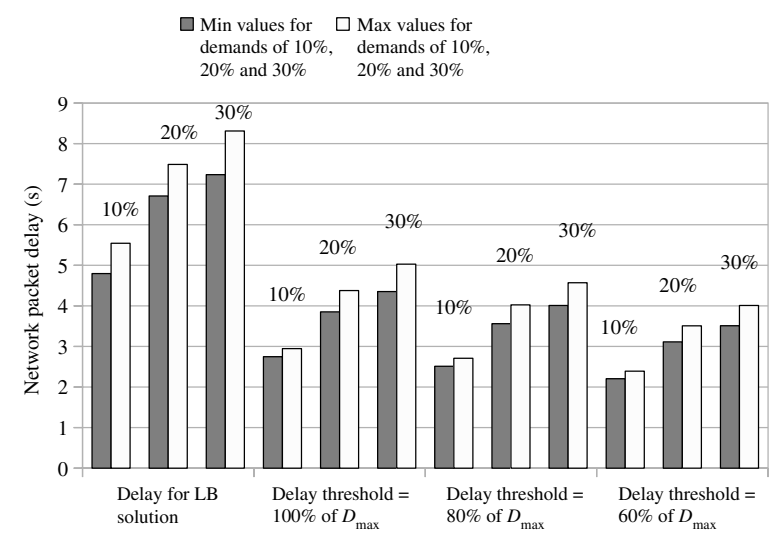

Fig. 8. Minimum and maximum network packet delays among all topology and gateway scenarios generated.

able to reduce the average packet delay, with negligible energy cost increases and without sacrificing network utilization, in many scenarios. The algorithm proved to be computationally efficient and scalable. Therefore, we can say that the proposed approach can be used for planning and design of QoS-aware energy-efficient FiWi access networks.

\section{ACKNOWLEDGMENTS}

This work was supported by FCT (Foundation for Science and Technology) of Portugal within CEOT (Center for Electronic, Optoelectronic and Telecommunications).

\section{REFERENCES}

[1] R. Bolla, R. Bruschi, F. Davoli, and F. Cucchietti, "Energy efficiency in the future Internet: a survey of existing approaches and trends in energy-aware fixed network infrastructures," IEEE Commun. Surv. Tutorials, vol. 13, no. 2, pp. 223-244, 2011.

[2] S.-W. Wong, L. Valcarenghi, S.-H. Yen, D. R. Campelo, S. Yamashita, and L. Kazovsky, "Sleep mode for energy saving PONS: Advantages and drawbacks," in IEEE GLOBECOM Workshops, 2009.

[3] B. Rengarajan, G. Rizzo, and M. Ajmone, "Bounds on QoSconstrained energy savings in cellular access networks with sleep modes," in 23rd Int. Teletraffic Congress, 2011, pp. 47-54.
[4] P. Chowdhury, M. Tornatore, S. Sarkar, and B. Mukherjee, "Building a green wireless-optical broadband access network (WOBAN)," J. Lightwave Technol., vol. 28, no. 16, pp. 2219-2229, Aug. 2010.

[5] C. Lange and A. Gladisch, "On the energy consumption of FTTH access networks," in IEEE OFC, 2009.

[6] J. Coimbra, G. Schütz, and N. Correia, "Forwarding repeated game for end-to-end QoS support in fiber-wireless access networks," in IEEE GLOBECOM, 2010.

[7] R. Jurdak, A. G. Ruzzelli, and G. M. P. O'Har, "Radio sleep mode optimization in wireless sensor networks," IEEE Trans. Mobile Comput., vol. 9, no. 7, pp. 955-968, July 2010.

[8] R. Gaudino, D. Cárdenas, M. Bellec, B. Charbonnier, N. Evanno, P. Guignard, S. Meyer, A. Pizzinat, I. Möllers, and D. Jäger, "Perspective in next-generation home networks: Toward optical solutions," IEEE Commun. Mag., vol. 48, no. 2, pp. 39-47, Feb. 2010.

[9] S. Sarkar, D. Dixit, and B. Mukherjee, "Hybrid wireless-optical broadband-access network (WOBAN): A review of relevant challenges," J. Lightwave Technol., vol. 25, no. 11, pp. 3329-3340, Nov. 2007.

[10] L. Chiaraviglio, M. Mellia, and F. Neri, "Reducing power consumption in backbone networks," in IEEE ICC, 2009.

[11] J. Baliga, R. Ayre, K. Hinton, W. V. Sorin, and R. S. Tucker, "Energy consumption in optical IP networks," J. Lightwave Technol., vol. 27, no. 13, pp. 2391-2403, July 2009.

[12] I. Cerutti, L. Valcarenghi, and P. Castoldi, "Power saving architectures for unidirectional WDM rings," in IEEE OFC, 2009.

[13] R. Kubo, J.-i. Kani, H. Ujikawa, T. Sakamoto, Y. Fujimoto, N. Yoshimoto, and H. Hadama, "Study and demonstration of sleep and adaptive link rate control mechanisms for energy efficient 10G-EPON," J. Lightwave Technol., vol. 2, no. 9, pp. 716-729, Sept. 2010.

[14] Z. Wang, W. Yu, and L. Wang, "Energy saving mechanism based on double-way polling DBA algorithm and ONU sleep mode for EPON," in Int. Conf. on Electronic and Mechanical Engineering and Information Technology (EMEIT), 2011.

[15] S.-W. Wong, S.-H. Yen, P. Afshar, S. Yamashita, and L. G. Kazovsky, "Demonstration of energy conserving TDM-PON with sleep mode ONU using fast clock recovery circuit," in IEEE OFC, 2010.

[16] Y. Yan, S.-W. Wong, L. Valcarenghi, S.-H. Yen, D. R. Campelo, S. Yamashita, L. Kazovsky, and L. Dittmann, "Energy management mechanism for Ethernet passive optical networks (EPONs)," in IEEE ICC, 2010.

[17] J. Lee, C. Rosenberg, and E. Chong, "Energy efficient schedulers in wireless networks: Design and optimization," Mob. Netw. Appl., vol. 11, no. 3, pp. 377-389, June 2006.

[18] D. Qiao, M. C. Gursoy, and S. Velipasalar, "The impact of QoS constraints on the energy efficiency of fixed-rate wireless transmissions," IEEE Trans. Wireless Commun., vol. 8, no. 12, pp. 5957-5969, Dec. 2009.

[19] S. Sahni and T. Gonzalez, "P-complete approximation problems," J. Assoc. Comput. Mach., vol. 23, pp. 555-565, 1976.

[20] M. R. Garey and D. S. Johnson, Computers and Intractability: A Guide to the Theory of NP-Completeness. W. H. Freeman and Co., San Francisco, 1979.

[21] C. E. Jones, K. M. Sivalingam, P. Agrawal, and J. C. Chen, "A survey of energy efficient network protocols for wireless networks," Wireless Networks, vol. 7, no. 4, pp. 343-358, July 2001.

[22] F. A. Onat and I. Stojmenovic, "Generating random graphs for wireless actuator networks," in IEEE Int. Symp. on a World of Wireless, Mobile and Multimedia Networks, June 2007, pp. 1-12. 